\title{
Introduction to Machine Learning for Digital Library Applications
}

\author{
Rodney D. Nielsen \\ Human Intelligence and Language \\ Technologies Lab \\ Computer Science \& Engineering, UNT \\ Denton, TX 76207, USA \\ Rodney.Nielsen@UNT.edu
}

\begin{abstract}
This tutorial begins with an overview of the major branches of machine learning (ML) and then provides more thorough coverage of deep neural networks. It covers key concepts, tools, experimental methods, applications, evaluation measures and associated issues for supervised learning (regression and classification), unsupervised learning (clustering and dimensionality reduction), semi-supervised and active learning (which combine the former approaches), and reinforcement learning. The deep neural network discussion covers convolutional neural networks (CNNs), recurrent neural networks (RNNs), word embeddings and related techniques. The discussion will be grounded on digital library (DL) - related applications and will highlight issues, techniques and tools associated with processing big data.
\end{abstract}

\section{CCS CONCEPTS}

- Computing methodologies $\rightarrow$ Machine Learning • Information systems $\rightarrow$ Digital libraries and archives;

\section{KEYWORDS}

machine learning, digital libraries, deep neural networks, tutorial

ACM Reference format:

Rodney D. Nielsen. 2018. Introduction to Machine Learning for Digital Library Applications. In Proceedings of The 18th ACM/IEEE Joint Conference on Digital Libraries, Fort Worth, Texas USA, fune 3-7 2018 (FCDL'18), 2 pages.

https://doi.org/10.1145/3197026.3201780

\section{INTRODUCTION}

Highlights of this tutorial include an emphasis on applications of machine learning (ML) in the digital libraries (DL) space, and an emphasis on deep neural networks, which have largely taken over vision and image processing (convolutional neural networks: CNNs) and analytics of sequence data (recurrent neural networks: RNNs)

Permission to make digital or hard copies of part or all of this work for personal or classroom use is granted without fee provided that copies are not made or distributed for profit or commercial advantage and that copies bear this notice and the full citation on the first page. Copyrights for third-party components of this work must be honored. For all other uses, contact the Owner/Author.

fCDL'18, fune 3-7, 2018, Fort Worth, TX, USA

(C) 2018 Copyright is held by the owner/author(s).

ACM ISBN 978-1-4503-5178-2/18/06.

DOI: https://doi.org/10.1145/3197026.3201780
It will not just describe algorithms, but will cover good experimental design methodology and evaluation to ensure your results generalize well to new (production) data. It will also cover appropriate task-dependent evaluation measures.

\section{TUTORIAL DESCRIPTION}

\subsection{Duration}

Half day

\subsection{Target Audience}

Beginner to intermediate: No prior experience or knowledge of ML will be required, but those at the intermediate level in $\mathrm{ML}$ who have little or no experience with deep learning, CNNs, RNNs, Big Data, and or DL applications, will also benefit.

Educators, students, scientists, managers and advisors: This tutorial will be beneficial for those interested in applying ML, particularly in the area of text analysis and DL, and for educators and managers involved in ML-related decision making and advising.

Expected number of participants: $\sim 20$

\subsection{Learning Objectives}

Given a problem statement, you will develop the skills to:

- Recognize whether and where machine learning techniques are applicable,

- Determine which set of the ML algorithms covered in the course are applicable,

- Design a machine learning application component,

- Design and conduct appropriate machine learning experiments,

- Analyze and properly describe the outcome of ML experiments, and

- Extract key information from literature.

\subsection{Outline}

This tutorial is comprised of two parts: 1) a broad overview of ML's categories, associated algorithms, tools, methods and issues, and 2) an introduction to deep neural networks. It will cover a number of machine learning topics, issues, and applications at varying levels, such as the following. (Core ML 
topics are in regular font; cross-cutting topics are italicized; and DL-related applications are underlined - prototypical and particularly good examples have a solid underline.)

- Overview of ML and its major categories

- $\quad$ Supervised Learning

$$
\begin{array}{ll}
\circ & \text { Tools } \\
\circ & \text { Regression }
\end{array}
$$

- Document quality assessment in curation

- Experimental Methods

- Evaluation Measures

- Classification

- Logistic Regression

- Spam detection

- Nearest Neighbor

- Authorship attribution

- Experimental Methods

- Evaluation Measures

- Support Vector Machines

- Decision Trees

- Naïve Bayes

- Document classification

- Unsupervised Learning

○ Clustering

- $k$-means

- Document clustering

- Experimental Methods

- Evaluation Measures

- Expectation Maximization

- Hierarchical Agglomerative Clustering

- Topic Modeling

- Document indexing

- Keyword extraction

- Dimensionality Reduction

- Principle Components Analysis

- Latent Semantic Analysis

- Information retrieval

- $\quad$ Active Learning
- Experimental Methods
- Evaluation Measures
- Genre classification

- $\quad$ Semi-supervised Learning
○ Self Training
- Word sense disambiguation
- Experimental Methods
- Evaluation Measures
- Co-Training
- Co-EM
- Document classification

- $\quad$ Reinforcement Learning

- Experimental Methods

- Evaluation Measures

- Curation
Part II:

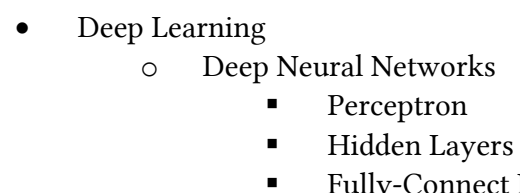

- Fully-Connect Feedforward NNs

- Document classification

- What's new?

- Experimental Methods

- Evaluation Measures

- Word Embeddings

- Nearly all text analytics

- Convolutional Neural Networks

- Image classification

- Recurrent Neural Networks

- Authorship attribution

- Article title generation

- Learning from Big Data

\section{BRIEF BIOGRAPHY}

Rodney Nielsen is an Associate Professor in the Department of Computer Science and Engineering at the University of North Texas. He received his Ph.D. from the University of Colorado at Boulder in 2008, where he went on to work as a Research Scientist in the Institute of Cognitive Science and adjunct in the Department of Computer Science. He was also a Research Scientist at Boulder Language Technologies and a Research Collaborator at Mayo Clinic. His research interests include ML, NLP, Cognitive Science and their application to companion healthcare robots for the elderly, educational technology, and health informatics. He has over 70 peer-reviewed research publications, has sat on related advisory boards, expert panels, shared task \& workshop organization boards, journal review panels, and numerous conference program committees. In addition to being the PI of federal grants totaling close to $\$ 4$ million, he was the lead R\&D designer and or proposal author of the artificial intelligence component of industrial contracts totaling nearly $\$ 8$ million, including one which won a AAAI Innovative Applications of Artificial Intelligence award.

\section{ACKNOWLEDGMENTS}

Thanks go to support by the Institute of Education Sciences, U.S. Department of Education, Grant R305A120808 to University of North Texas, the NSF Graduate Research Fellowship Program under Grant 1144248, and the NSF under Grant 1262860. Any opinions, findings, and conclusions or recommendations expressed in this material are those of the author(s) and do not necessarily reflect the views of the National Science Foundation or the Institute of Education Sciences. 